\title{
INVESTIGATING THE PREDICTIVE CAPACITY OF EULERIAN CFD TO MODEL LONG-DURATION BLAST LOADS ON FINITE CROSS-SECTION GEOMETRIES
}

\author{
JACK DENNY \& SIMON CLUBLEY \\ Blast Engineering Research Group, Faculty of Engineering and the Environment, \\ University of Southampton, UK.
}

\begin{abstract}
Typically defined by positive pressure durations over $100 \mathrm{~ms}$, long-duration blasts can generate dynamic pressures (blast winds) capable of exerting damaging drag loads on slender structural elements such as columns. With limited availability of appropriate drag coefficients for specific structural geometries or different section orientations, Computational Fluid Dynamics (CFD) can provide a valuable tool for calculating blast interaction and loading on user-specified geometries. Commercially available CFD programs or 'hydrocodes' with shock wave modelling capabilities remain based on solving the inviscid Euler equations. The ability to analyse long-duration blasts is still not confidently offered however, with no prior studies examining the accuracy of modelling interaction with relatively much smaller, finite geometries. This remains particularly challenging due to large wavelengths and time durations inherent to long-duration blasts, usually limited by impractical solution domains and computing resource. This paper presents a comparative investigation between numerical simulations and experimental results to assess the predictive capability of Eulerian CFD as a tool for calculating long-duration blast drag loading on an intricate I-section geometry from different angles of incidence. Calculated pressure-time histories on exposed geometry surfaces demonstrated good agreement although reduced accuracy and under-prediction occurred for shielded surfaces manifesting as overestimated net translational loading. Numerical discrepancies were attributed to the inviscid Euler equations underpinning the CFD solver, limiting accuracy when resolving complex aerodynamic flows at bluff I-section orientations. Results of this study provide new understanding and awareness of the numerical capability and limitations of using CFD to calculate long-duration blast loads on intricate geometries.
\end{abstract}

Keywords: Blast loading, Drag loading, Eulerian CFD, Long-duration, Multi-axis

\section{INTRODUCTION}

Long-duration blast waves are typically defined by positive pressure durations over $100 \mathrm{~ms}$, developing in later stages of shock wave propagation i.e. in the 'far field' from large-scale explosions. In the modern world, these are most commonly caused by hydrocarbon vapour cloud explosions (VCE) at petrochemical facilities due to the nature in which volatile hydrocarbons are stored, equating to large amounts of potential energy. Notable examples include the 2005 'Buncefield Disaster', exhibiting an estimated TNT equivalence of 105-250T [1, 2]. The prevalence of such accidents highlight a growing need to accurately model structural loading resulting from long-duration blasts.

Long-duration blasts are extremely powerful, generating non-trivial dynamic pressures (blast winds) capable of exerting significant drag loads on slender structural elements from various angles of incidence depending on the location of detonation. Correctly characterising blast loading on structural elements is essential for deriving reliable structural response solutions. Calculating time varying blast pressures on component surfaces of structural elements can be complex, particularly when considering different angles of incidence or intricate geometry features. Engineers therefore resort to simplifications and expedite the calculation of translational drag loading by incorporating drag coefficients which attempt to encapsulate complex aerodynamic processes within a single modifier. The concept of modifying the 
product of dynamic pressure and projected area by a dimensionless drag coefficient $C_{D}$, has been universally adopted in blast design guidance and literature [3-6]. The prevalence of blast drag coefficients in open literature is limited, particularly when considering specific geometries such as structural I-sections. Drag coefficients proposed in literature also demonstrate inconsistency and are typically single values, lacking provision for different angles of incidence.

In such cases where simplified empirical methods are inadequate, Computational Fluid Dynamics (CFD) may be the only satisfactory approach for calculating blast loading on user-specified geometries. CFD is a well-established numerical method utilising the physical equations of fluid dynamics to simulate the propagation of fluid flow through a domain and around objects. Fluid dynamics are the fundamental physical equations underpinning CFD and are based on the conservation of mass (continuity), momentum and energy, collectively known as the Navier-Stokes equations [3, 4, 7]. Simplifications of the Navier-Stokes equations are known as the Euler equations, in which the fluid is assumed to be 'inviscid' (i.e. an ideal fluid, in which kinematic and dynamic viscosity, $\mu$ and $v$ are zero) and no heat conduction exists [4]. Such inviscid flow assumptions are appropriate for high-speed external flows around streamlined bodies or 'free-stream' flows at sufficient distance from a body [8]. Inviscid flows neglect viscous shear stresses and are assumed irrotational (i.e. no vorticity), although such approximation is not valid within boundary layers or wake regions [8].

Schraml \& Hisley [9] investigated the accuracy of both Euler and Navier-Stokes flow solvers to model short-duration blast interaction with a 2D square target with comparison to experimental measurements performed inside a shock tube. Results of the study demonstrated that the inviscid solver was less accurate than the viscous (Navier-Stokes) solver for resolving pressure histories on the side surfaces of the square target during the drag phase [9]. While viscous Navier-Stokes solvers have potential to resolve complex aerodynamic flows pertaining to the drag phase, no such commercial codes offering shock wave modelling are openly available. Commercially available CFD programs or 'hydrocodes' with shock wave modelling capabilities remain based on solving the Euler equations such as Air3D [10] and ANSYS Autodyn [11]. With the majority of contemporary blast research concentrated on short-duration explosions where drag loading is inconsequential, such inviscid Euler solvers are adequate and have demonstrated satisfactory accuracy in prior blast research [12-14]. No studies have assessed the accuracy and reliability of Eulerian CFD for modelling long-duration blast interaction with small, intricate geometries where drag loading is non-trivial.

Modelling long-duration blasts with CFD is still not confidently offered. Such blasts inherently involve long positive pressure durations $\left(\mathrm{t}^{+}>100 \mathrm{~ms}\right)$, large wavelengths and standoff distances which readily give rise to impractical solution domains, simulation times and computational resource. The challenging spatial scale of the problem is exacerbated when attempting to model interaction with much smaller, intricate geometries that require high resolution meshes. In such cases, careful employment of mesh-rezoning techniques is essential to address the relative spatial scales and manage the associated computational expense.

This paper presents a comparative investigation between numerical simulations and experimental results to assess the capability of Eulerian CFD as a tool for calculating long-duration blast drag loading on an intricate I-section geometry from different angles of incidence. Blast loading on I-shape geometries presents a particularly challenging scenario, giving rise to complex blast interaction due to axis sensitivity and intricate geometric features (web and flanges). 


\section{METHODOLOGY}

\subsection{Experimental Methodology}

Long-duration blast waves were generated using the national Air Blast Tunnel (ABT) facility at MoD Shoeburyness, UK (Fig. 1a). A driver charge located in a $1.8 \mathrm{~m}$ diameter section and the long increasing diameter of the tunnel effectively re-shapes the shock into a substantially longer wavelength with characteristics of a near-planar long-duration blast. For each experimental trial, blast waves with consistent parameters were sought inside the $10.2 \mathrm{~m}$ section, including a peak overpressure of $\mathrm{p}_{\mathrm{i}} \approx 55 \mathrm{kPa}$ and positive phase duration of $\mathrm{t}^{+} \approx 150 \mathrm{~ms}$.

Four long-duration blast trials were conducted in the ABT to measure surface blast pressures and loading on a $3.00 \mathrm{~m}$ steel (S275) UKC 203x203x46 I-section column. Four angles of incidence were investigated by aligning the column at orientations of $0,30,60$ and 90 degrees to the blast propagation inside the ABT (Fig. 1b). The column was fixed to the ground in a cantilever configuration and designed for minimal elastic response, allowing repeat firings at different I-section orientations (Fig. 1c). A bespoke adjustable base plate design provided a fully fixed support condition that allowed the section to be rotated in-situ between trials to achieve specified orientations (Fig. 1d). The I-section was instrumented with eight Endevco $8515 \mathrm{C}-50$ pressure transducers secured to the centre point of each surface at

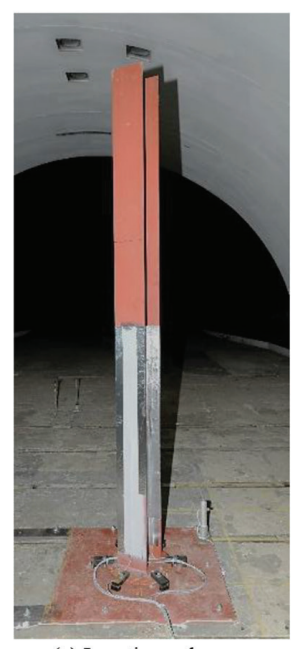

(c) I-section column cantilever configuration

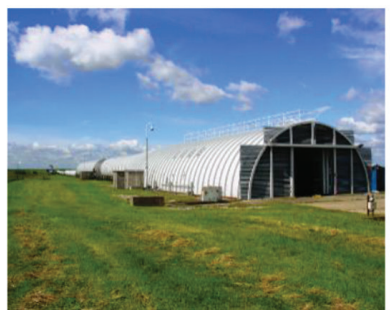

(a) The Air Blast Tunnel (ABT), MOD

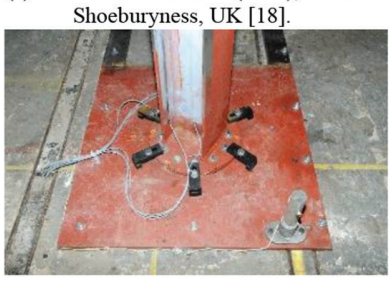

(d) Circular baseplate design

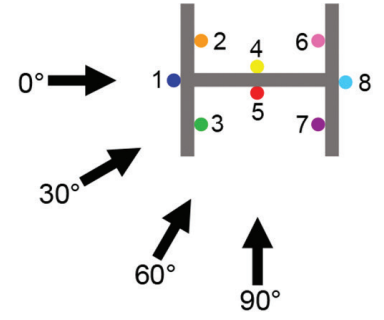

(b) Multi-axis interaction and gauge numbering convention

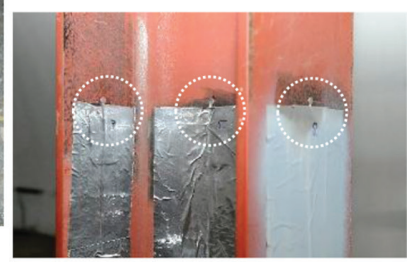

(e) Surface pressure transducers

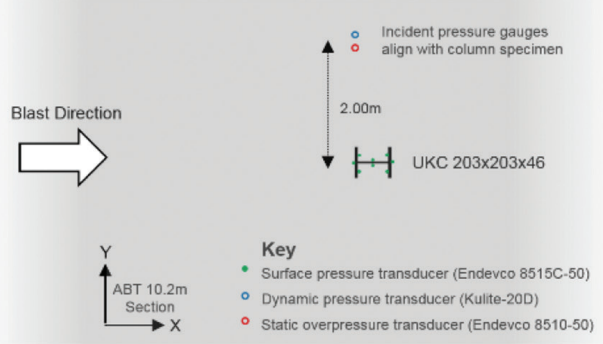

(f) Plan view schematic of long-duration blast trials configuration inside the ABT.

Figure 1: Long-duration blast trials setup inside the Air Blast Tunnel (ABT). 
mid-height to measure blast pressures exerted on respective column surfaces (Fig. 1b and e). The incident blast environment was measured using Endevco 8510-50 static overpressure gauges and Kulite-20D dynamic pressure gauges (Fig. 1f).

\subsection{Numerical Methodology}

Eulerian CFD models were developed to calculate surface pressure-time histories on respective I-section surfaces at the four axes corresponding to the experimental trials. ANSYS Autodyn [11] was utilised primarily due to its domain 'rezoning' capabilities. Importantly, this controlled computational expense by enabling a locally high-resolution mesh to be defined within a much larger coarse mesh, suitable for representing I-section geometry. Numerical modelling was performed in two stages:

1. A one-dimensional (1D) CFD analysis of a spherical free-air explosion was performed to generate a long-duration blast wave with blast parameters equivalent to the experimental incident blast conditions inside the ABT.

2. Two-dimensional (2D) Eulerian CFD analyses were performed by 'remapping' the incident blast wave (from prior 1D analysis) to propagate at specified angles across a planar domain to interact with a rigid I-section geometry corresponding to the experiments. Surface pressure gauges assigned to the rigid I-shape geometry measured pressure-time histories for comparison to experimental results.

\subsubsection{D CFD: Incident Blast Wave}

The experimental incident blast wave was simulated by modelling an assumed spherical freeair explosion of a certain explosive charge mass and standoff distance that generated equivalent blast wave parameters (Fig. 2a and b). Detonation of a spherical TNT charge and subsequent blast wave propagation in air was modelled using a radially symmetric onedimensional (1D) wedge domain, comprising 25,000 10mm cells.

A sphere of TNT material was assigned at the apex of the wedge domain containing atmospheric air elements (Fig. 2c). Explosive TNT material was modelled using the JonesWilkins-Lee (JWL) equation of state (EOS) [15] and air was modelled as an ideal gas with an ambient pressure of $101.33 \mathrm{kPa}$ by specifying an internal energy of $2.068 \times 10^{5} \mathrm{~mJ} / \mathrm{mm}^{3}$. Initial temperature, density and adiabatic constant (specific heat ratio) $\Upsilon$ of air cells were set to $288^{\circ} \mathrm{K}\left(15^{\circ} \mathrm{C}\right), 1.225 \mathrm{mg} / \mathrm{cm}^{3}$ and 1.4 respectively. The multi-material Euler-Godunov solver was used to model both the detonation of explosive material and subsequent shock wave propagation through air.

Calculations based on the empirical work of Kingery \& Bulmash [16] were used to approximate the required TNT charge mass and standoff distance to generate an incident blast wave

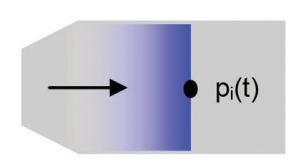

(a) Experimental planar incident blast wave in the ABT

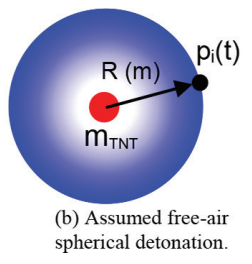

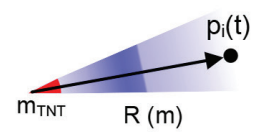

(c) 1D wedge domain schematic.

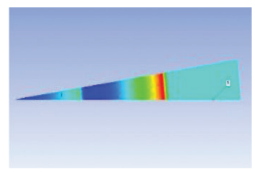

(d) 1D CFD radial blast propagation.

Figure 2: A spherical free-air explosion was modelled to generate equivalent incident longduration blast environment to the ABT. 
equivalent to that measured in the ABT. Through an iterative process, a charge mass of 268T and standoff distance of $220 \mathrm{~m}$ was required. CFD analysis was performed until the shock front propagated to the required standoff distance and then saved as a remap file to be utilised as initial conditions for subsequent $2 \mathrm{D}$ analyses.

\subsubsection{Two-Dimensional CFD: I-section Blast Interaction}

Two-dimensional (2D) CFD analyses were performed to model blast interaction with the I-section geometry; uniform loading along the column height was assumed corresponding to a planar blast wave generated within the ABT. Preliminary modelling identified the domain had to be large enough to accommodate the entire blast wavelength before and after the cross-section. Transmissive "flow out" boundary conditions were assigned to all sides to prevent reflection to ensure the interaction of a single, incident blast wave with the I-section geometry. These boundary conditions were not entirely effective however, with some localised reflection occurring at the domain sides. Dimensions of the domain therefore had to be sufficiently large to prevent any boundary perturbations from interfering with the regions of interest, particularly for oblique axis blast propagation.

Informed by sensitivity studies, a 2D square domain with dimensions of $200 \mathrm{~m}$ x 200m was defined comprising 1 million cells to achieve maximum accuracy at manageable computational expense. Mesh re-zoning achieved a locally fine mesh comprising $5 \mathrm{~mm}$ cells at the centre of the 2D domain, allowing void 'un-used' cells to be specified to reasonably represent the I-section geometry tested in experimental trials (Fig. 3).

Reflective boundaries were assigned to void cells at geometry edges, assuming a perfectly rigid column. Pressure monitoring points were assigned in air cells adjacent to the centre point of each cross section edge to record surface pressure-time histories, corresponding to surface pressure transducers (Fig. 3). Additional pressure monitoring points were specified $2.00 \mathrm{~m}$ from the column geometry to record the local incident free-field blast parameters

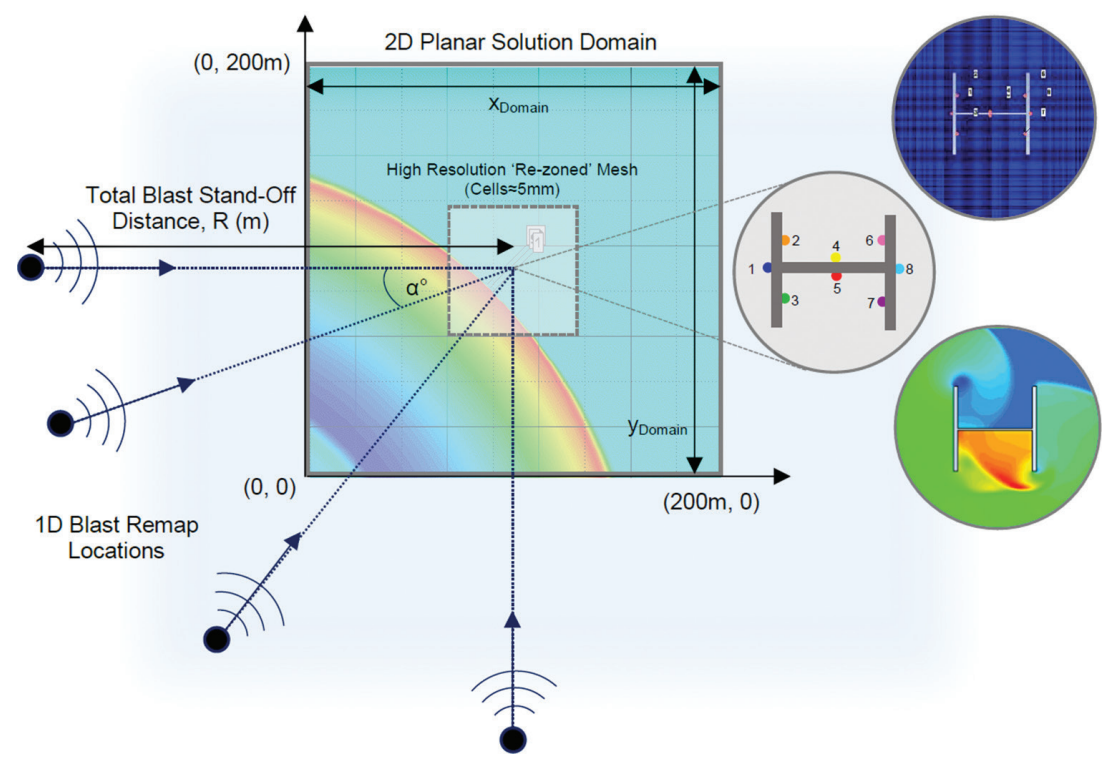

Figure 3: 2D domain setup and 1D remap locations to achieve multi-axis blast interaction. 
consistent with the experimental setup. Coordinate positions of 1D remap locations relative to the column geometry were determined using trigonometric relations to satisfy the standoff distance and angle of incidence for blast propagation across the planar domain (Fig. 3).

The planar domain was defined and filled with air modelled as an ideal gas. A single material, Euler Flux Corrected Transport (FCT) solver was adopted as only the blast wave (air) itself required modelling. This solver has the advantage of being fast and efficient, specifically developed for blast applications $[17,18]$. Blast waves from 1D analyses were remapped into the $2 \mathrm{D}$ domain and simulated for a duration of $\mathrm{t}=170 \mathrm{~ms}$, allowing interaction with the I-section geometry for the full positive phase duration. Pressure data was recorded at increments of $0.2 \mathrm{~ms}$, sufficiently resolving overpressure histories while maintaining manageable data storage. Simulation run times varied for different orientations simulated, although typically required $46 \mathrm{CPU}$ hours and $\approx 10 \mathrm{~GB}$ RAM.

\section{RESULTS \& DISCUSSION}

\subsection{Modelling the Incident Blast Environment}

Numerical incident overpressure-time histories and cumulative impulse curves show good agreement with experimental data in terms of peak overpressure, positive phase duration and total impulse (Fig. 4). The CFD model overpressure-time history represents a typical Friedlander profile whereas the experimental measurements, ignoring inevitable data noise, demonstrate three discontinuities throughout the positive phase duration (Fig. 4). Such nonideal behaviour appears in archival pressure records, indicating that this pattern is a possible artefact of operating the ABT facility at maximum power [19].

Peak incident blast overpressure demonstrated good agreement with experimental measurements, within $1.0 \%$ on average for the four orientations modelled (Table 1a). Total incident impulses calculated by the CFD model were slightly higher than experimental results with an average discrepancy of $1.4 \%$ across four trials, representing a typical impulse error of the order of $\approx 40 \mathrm{kPa}$.ms (Table $1 \mathrm{~b}$ ). Results show that a well-replicated incident blast wave consistent with the experiments was generated, suitable for extending analysis to subsequent interaction with a rigid I-section geometry.

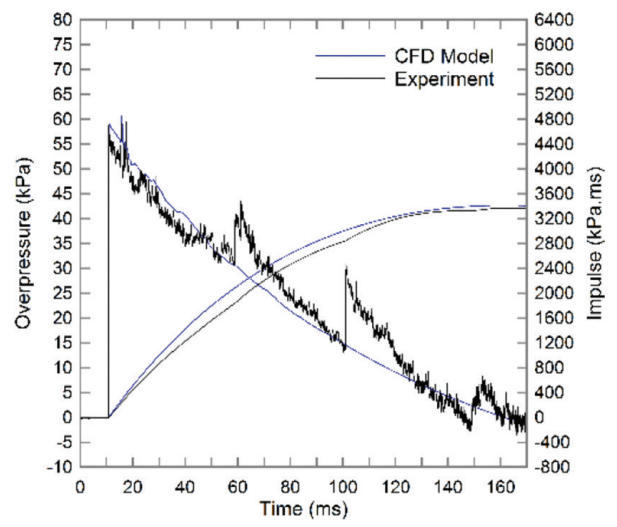

Figure 4: Incident overpressure-time histories and impulse. 
Table 1: Experimental and CFD model incident blast wave parameters.

(a) Peak incident blast overpressure.

\begin{tabular}{cccccc}
\hline & \multicolumn{5}{c}{ Peak Incident Overpressure, $\mathrm{p}_{\mathrm{i}}(\mathrm{kPa})$} \\
\cline { 2 - 6 } & $0^{\circ}($ Trial 1) & $30^{\circ}($ Trial 2) & $60^{\circ}$ (Trial 3) & $90^{\circ}$ (Trial 4) & Mean \\
\hline Experiment & 59.1 & 58.4 & 58.6 & 58.5 & 58.7 \\
CFD Model & 58.0 & 59.4 & 58.6 & 58.2 & 58.6 \\
\% Error & $-1.9 \%$ & $1.7 \%$ & $0.0 \%$ & $-0.5 \%$ & $1.0 \%$ \\
\hline
\end{tabular}

(b) Total incident impulse.

\begin{tabular}{cccccc}
\hline & \multicolumn{5}{c}{ Incident Total Impulse, $\mathrm{I}_{\mathrm{i}}(\mathrm{kPa} . \mathrm{ms})$} \\
\cline { 2 - 6 } & $0^{\circ}$ (Trial 1) & $30^{\circ}($ Trial 2) & $60^{\circ}$ (Trial 3) & $90^{\circ}$ (Trial 4) & Mean \\
\hline Experiment & 3377 & 3387 & 3327 & 3389 & 3370 \\
CFD Model & 3418 & 3414 & 3418 & 3421 & 3418 \\
\% Error & $1.2 \%$ & $0.8 \%$ & $2.7 \%$ & $0.9 \%$ & $1.4 \%$ \\
\hline
\end{tabular}

\subsection{I-Section Blast Interaction}

Surface overpressure-time histories calculated by CFD models were integrated over the positive phase duration to calculate total surface impulses (Table 2; Fig. 5). Despite a well-replicated incident blast wave, agreement between the CFD models and experimental results varied depending on the surface and I-section orientation considered. Surface impulse discrepancies between the results appear to correlate with surface exposure to the blast. For the purpose of analysis, 'exposed' or 'shielded' I-section surfaces are defined by total impulses exceeding or below the incident value, plotted in Fig. 5a and b respectively.

CFD models demonstrated comparably better agreement with experimental measurements for exposed I-section surfaces, particularly for orientations greater than $30^{\circ}$ i.e. once they become exposed (Fig. 5a). CFD analyses calculated surface total impulses with good agreement to experiments for surfaces 3,5 and 7 across the orientations investigated, with mean absolute percentage errors of $3.5 \%, 1.8 \%$ and $2.8 \%$ respectively (Table 2). As an example, pressure histories and cumulative impulse curves for surface 1 at the $60^{\circ}$ orientation display good agreement with the CFD model, under-predicting total impulse by only $0.9 \%$ (Fig. 6a; Table 2). Total impulse discrepancies between the CFD model and experiments were generally larger for shielded surfaces 2, 4, 6 and 8, with mean absolute percentage errors of $5.9 \%$, $5.2 \%, 5.6 \%$ and $12.8 \%$ respectively for the four orientations investigated (Fig. 5b; Table 2). This suggests that Eulerian CFD is less reliable at calculating pressure-time histories (and total impulse) on shielded surfaces of intricate geometries.

Larger discrepancies consistently occurred for surface 8, with a mean absolute percentage error of $12.8 \%$ across the four orientations investigated (Fig. 5b; Table 2). Models consistently calculated lower total impulse on surface 8 than measured in the experiments by the order of 200-500 kPa.ms depending on the orientation, representing percentage errors of 8.4-17.5\% (Table 2). The largest discrepancy occurred for surface 8 at the $30^{\circ}$ orientation where the CFD model under-predicted total impulse by 496kPa.ms (-17.5\%). Overpressure-time histories and 


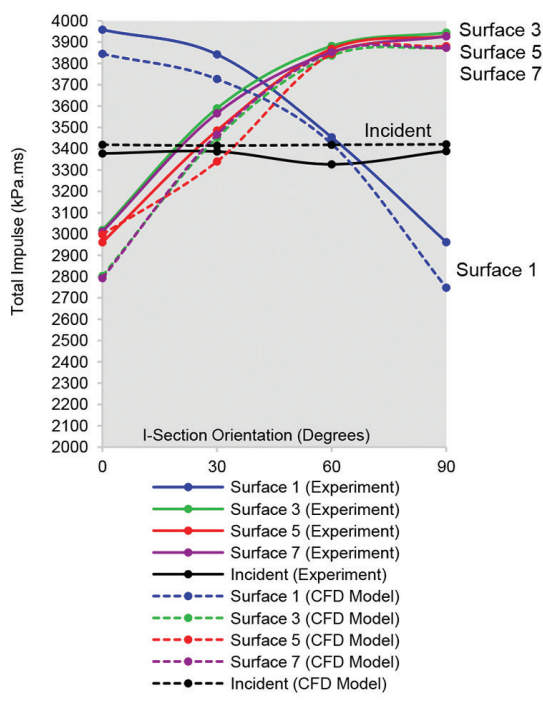

(a) Exposed Surfaces

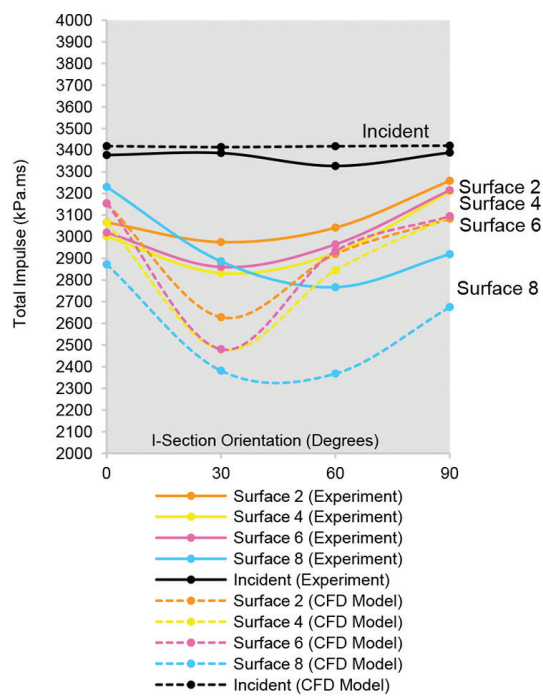

(b) Shielded Surfaces
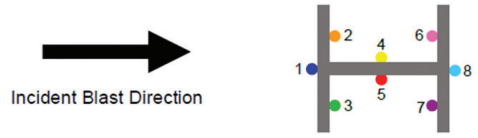

$0^{\circ}$

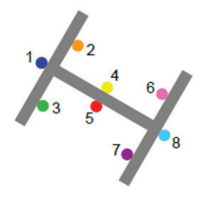

$30^{\circ}$

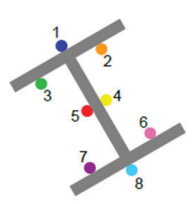

$60^{\circ}$

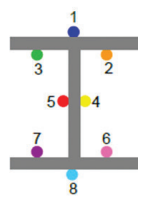

$90^{\circ}$

Figure 5: Surface total impulses measured experimentally and calculated by CFD models.

cumulative impulse curves calculated by the model and corresponding experimental measurements for surface 8 at the $30^{\circ}$ orientation are plotted in Fig. $6 \mathrm{~b}$. While peak overpressure and positive phase durations show good agreement, stagnation pressure histories beyond shockwave arrival results in relatively large cumulative and total impulse discrepancies (Fig. 6b). Distorted, oscillating stagnation pressures indicate the turbulent nature of the time-varying flow field in this location, characteristic of aerodynamic processes including shedding vortices, flow separation and low-pressure wake regions. The CFD model generally underestimated stagnation pressures, illustrated by diverging cumulative impulse curves beyond $t=50 \mathrm{~ms}$ in Fig. 6b.

Agreement between CFD models and experimental measurements appear to be governed not only by shielding (surface exposure), but also the presence of time-varying aerodynamic processes. Surface 4 at the $90^{\circ}$ I-section orientation for example, represents a fully shielded rear-facing surface yet peak overpressure, positive phase duration and total impulse exhibit fair agreement between the model and experiments (Fig. 6c). In this case, the CFD model underestimated surface total impulse by $114.3 \mathrm{kPa} . \mathrm{ms}(-3.6 \%)$, representing a relatively small error in comparison to other shielded surfaces (Table 2). Relatively better agreement for surface 4 at the $90^{\circ}$ orientation in comparison to surface 8 at the $30^{\circ}$ orientation may therefore be attributed to reduced flow separation or wake effects. These aerodynamic effects are not well simulated and likely to be symptomatic of the underpinning Euler solver.

Surface 6 presents a further example assessing the capability of Eulerian CFD to resolve stagnation pressure histories on shielded surfaces and the influence of aerodynamic processes. The CFD model under-predicted total impulse by $380 \mathrm{kPa} . \mathrm{ms} \mathrm{(-13.3 \% )}$ on surface 6 


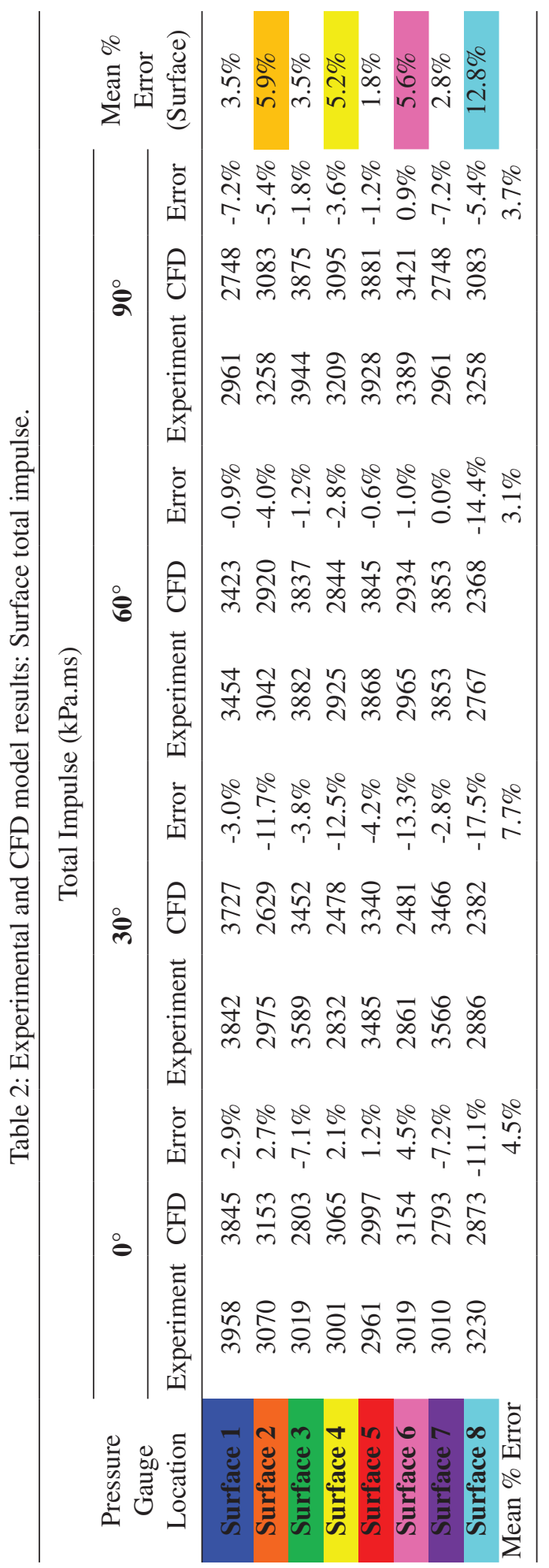



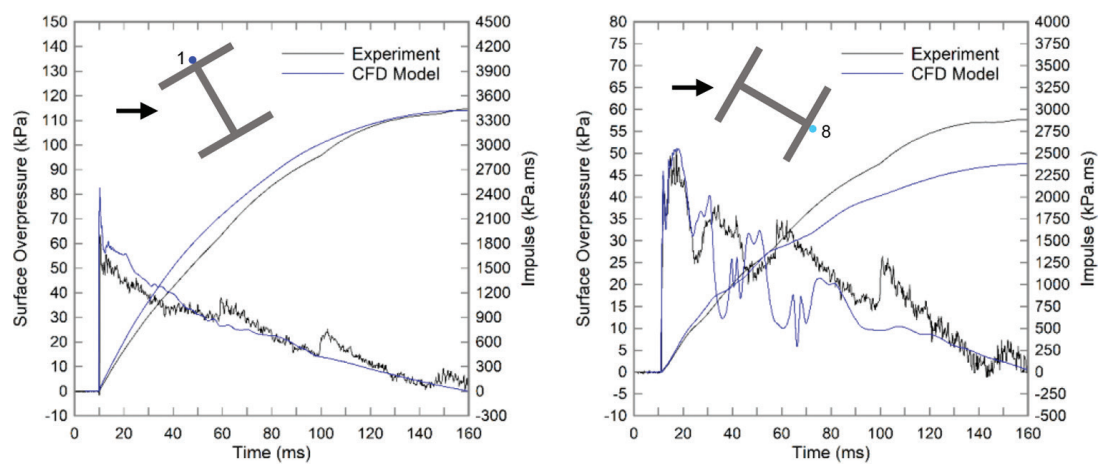

(a) Surface $1-60^{\circ}$

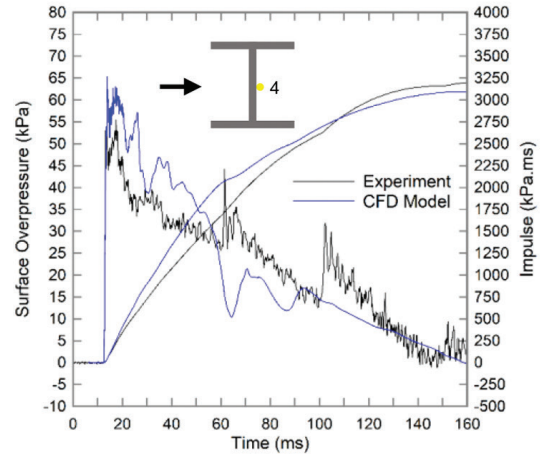

(b) Surface $8-30^{\circ}$

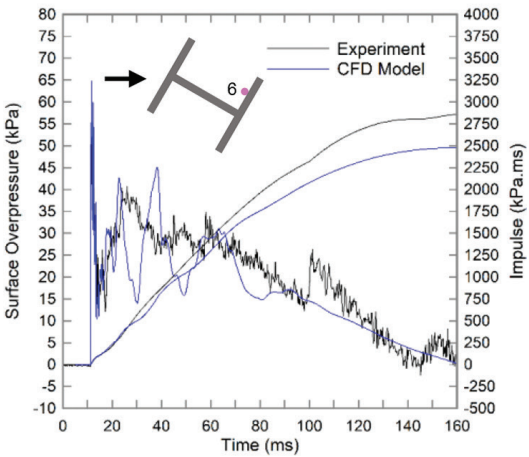

(d) Surface $6-30^{\circ}$

Figure 6: Experimental and CFD model surface pressure-time histories.

at the $30^{\circ}$ orientation (Table 2). For this case, the CFD model overpressure-time history exhibited an oscillating pressure profile generally below the measured experimental stagnation pressures, resulting in diverging cumulative impulse curves throughout the positive phase duration (Fig. 6d). Without consideration of advanced aerodynamics, flow separation originating at the top of the front flange at the $30^{\circ}$ orientation sheilds rear internal surfaces within a region of low-pressure wake that cannot be accurately simulated by the Eulerian CFD solver.

\subsection{Calculated Resultant Loading}

Surface pressure-time histories calculated by CFD models were multiplied by respective projected surface areas and resolved in the blast X-direction to calculate net force-time plots and cumulative X-impulse exerted on the I-section geometry (Fig. 7). Experimental net forcetime plots and cumulative impulse curves are also overlaid for comparison (Fig. 7).

Net force-time profiles calculated from CFD results visibly exceed those measured in experiments for I-section orientations of $30^{\circ}$ and $60^{\circ}$ (Fig. $7 \mathrm{~b}$ and c). Cumulative net impulse curves also exhibit steeper gradients for these orientations indicating that CFD analysis calculates a higher rate of net loading on the column geometry than measured in the experiments (Fig. 7b and c). CFD models of the orthogonal I-section orientations $\left(0^{\circ}\right.$ and $\left.90^{\circ}\right)$ demonstrate comparably better agreement in terms of cumulative impulse curves although these also exhibited noticeable deviation from the experimental results at a later time during the positive phase ( $\mathrm{t}>100 \mathrm{~ms}$ ) (Fig. 7a and $d$ ). 


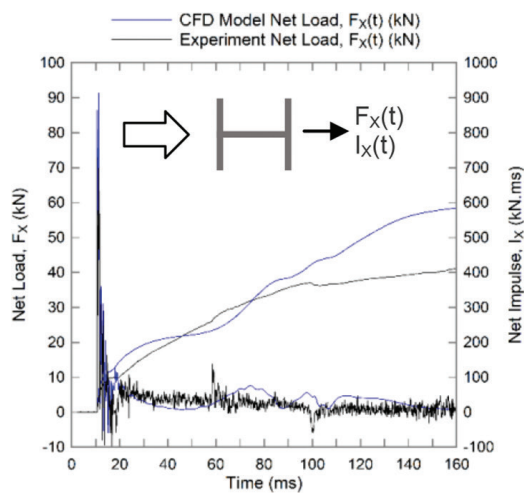

(a) $0^{\circ}$ Resultant X-Force-Time History

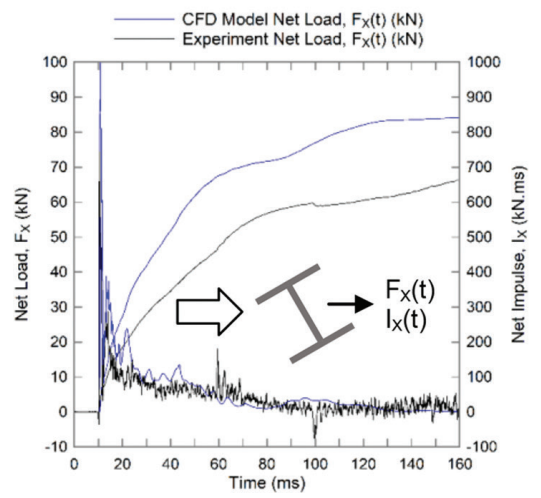

(c) $60^{\circ}$ Resultant X-Force-Time History

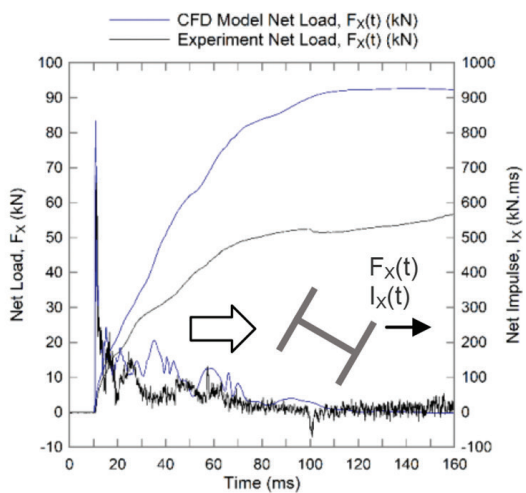

(b) $30^{\circ}$ Resultant X-Force-Time History

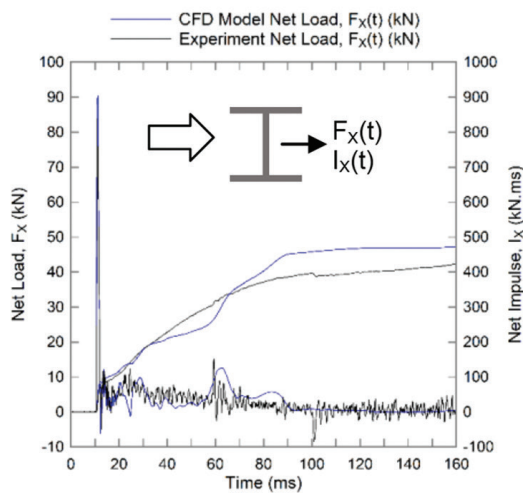

(d) $90^{\circ}$ Resultant X-Force-Time History

Figure 7: Net force-time histories and impulse: Experimental results vs. CFD models.

Total net impulses accumulated over the positive phase duration $\left(\mathrm{t}=\mathrm{t}_{\mathrm{a}}+150 \mathrm{~ms}\right)$ are tabulated in Table 3. For all orientations, net total impulses calculated using CFD analysis exceed experimental results with large discrepancies except for the $90^{\circ}$ orientation (Table 3). At I-section orientations of $0^{\circ}$ and $30^{\circ}$, net total impulse calculated from CFD analyses exceeded experimental values by $170 \mathrm{kN} . \mathrm{ms}(40.9 \%)$ and $356 \mathrm{kN} . \mathrm{ms}(62.3 \%)$ respectively (Table 3 ). Overall, CFD analyses over-predicted total and cumulative impulse resultant loading on the I-section geometry.

Table 3: Total net impulse (positive phase duration): Experimental results vs. CFD models.

\begin{tabular}{ccccc}
\hline & \multicolumn{4}{c}{ Total Net X-Impulse During Positive Phase Duration, $\mathrm{I}_{\mathrm{X}}(\mathrm{kN} . \mathrm{ms})$} \\
\cline { 2 - 5 } & $0^{\circ}$ & $30^{\circ}$ & $60^{\circ}$ & $90^{\circ}$ \\
\hline Experiment & 415 & 567 & 662 & 423 \\
CFD Model & 585 & 923 & 840 & 472 \\
$\%$ Error & $40.9 \%$ & $62.3 \%$ & $26.9 \%$ & $11.6 \%$ \\
\hline
\end{tabular}




\section{CONCLUSIONS}

Numerical modelling results were compared to corresponding experimental measurements to assess the predictive capacity of inviscid Eulerian CFD as a tool for calculating long-duration blast drag loading on intricate cross-section geometries. Analysis of model results showed that Euler CFD can reliably calculate surface stagnation pressure-time histories on exposed surfaces of a finite cross section geometry subjected to a long-duration blast. For surfaces directly exposed to blast, total impulse error between the numerical models and experiments were less than $3.5 \%$ on average throughout the orientations investigated. CFD models generally underestimated stagnation pressures and impulses on shielded, rear-facing geometry surfaces despite the numerical incident blast wave exhibiting good agreement to the experimental environment. With closer inspection of specific surfaces, larger discrepancies occurred in locations where flow separation, turbulence and wake regions have significant influence. For these cases, Euler CFD exhibits less accuracy for calculating stagnation pressure profiles due to incorrectly resolving complex aerodynamic effects due to limitations of the underpinning inviscid flow solver and no numerical turbulence model.

Underestimation of stagnation pressures on rear-facing, shielded geometry surfaces manifested as overestimated resultant loading, notably exceeding experimental results. Resultant blast loading was most significantly overestimated by CFD analyses at bluff, oblique I-section orientations where complex aerodynamic effects were predominant. This study provides new awareness toward the capability and limitations of inviscid hydrocodes for long-duration blast loads on intricate geometries where drag loading is predominant. In the absence of verified drag coefficients for specific geometries or orientations, Eulerian CFD analysis provides a conservative method for characterising blast loading on intricate user-specified geometries, although reduced accuracy can be expected where complex aerodynamic processes have significant influence.

\section{ACKNOWLEDGEMENTS}

The authors would like to express gratitude to the UK Ministry of Defence for allowing use of the blast testing facilities at MoD Shoeburyness. All data obtained whilst using these facilities remains the property of the UK MoD. The authors wish to thank the UK EPSRC and AWE plc for financial support.

\section{REFERENCES}

[1] Steel Construction Institute, "Buncefield Explosion Mechanism Phase 1 (Volumes 1 and 2)," Ascot, 2009.

[2] Atkinson, G. \& Cusco, L., Buncefield: a violent, episodic vapour cloud explosion. Process Safety and Environmental Protection, 89(6), pp. 360-370, 2011. https://doi.org/10.1016/j.psep.2011.06.019

[3] Smith, P.D. \& Hetherington, J.G., Blast and Ballistic Loading of Structures, CRC Press, Boston, FL, 1994.

[4] Mays, G. \& Smith, P., Blast Effects on Buildings, 1st edn. Thomas Telford Limited, London, 1995.

[5] Krauthammer, T., Modern Protective Structures, CRC Press, Boston, FL, 2008.

[6] US Department of Defense (DoD), "UFC 3-340-02, 'Structures To Resist The Effects Of Accidental Explosions,", Washington, DC, 2008.

[7] Anderson, J.D., Computational Fluid Dynamics: The Basics with Applications, 3rd Edn, Springer, New York, NY, 2009. 
[8] Craft, T.J., Inviscid Flows - Fluid Mechanics, The University of Manchester, Manchester, 2010.

[9] Schraml, S.J. \& Hisley, D.M., Euler and Navier-Stokes Simulations of Shock Wave Interaction With a Generic Block Target (ARL-TR-848), 1995.

[10] Rose, T.A., Air3D Version 9.0 - Computational Tool for Airblast Calculations. Engineering Systems Department, Cranfield University, 2006.

[11] Century Dynamics, "AUTODYN.” 2011.

[12] Chapman, T.C., Rose, T.A. \& Smith, P.D., Blast wave simulation using AUTODYN2D: a parametric study. International Journal of Impact Engineering, 16(5-6), pp. 777-787, 1995.

https://doi.org/10.1016/0734-743x(95)00012-y

[13] Ballantyne, G.J., Whittaker, A.S., Dargush, G.F. \& Aref, A.J., Air-blast effects on structural shapes of finite width. Journal of Structural Engineering, 136(2), pp. 152-159, 2010. https://doi.org/10.1061/(asce)st.1943-541x.0000049

[14] Sherkar, P., Whittaker, A.S. \& Aref, A.J., Modeling the Effects of Detonations of High Explosives to Inform Blast-Resistant Design (Technical Report MCEER-10-0009), 2010.

[15] Lee, E.L., Hornig, H.C. \& Kury, J.W., Adiabatic Expansion of High Explosive Detonation Products (Report UCRL-50422), 1968.

[16] Kingery, C.N. \& Bulmash, G., Airblast parameters from tnt spherical air burst and hemispherical surface burst (Technical Report ARBRL-TR-02555), 1984.

[17] Fairlie, G.E., The numerical simulation of high explosives using AUTODYN-2D \& 3D, 1998.

[18] Børvik, T., Hanssen, A.G., Langseth, M. \& Olovsson, L., Response of structures to planar blast loads - a finite element engineering approach. Computers \& Structures, 87(9-10), pp. 507-520, 2009. https://doi.org/10.1016/j.compstruc.2009.02.005

[19] Clubley, S.K., Non-linear long duration blast loading of cylindrical shell structures. Engineering Structures, 59, pp. 113-126, 2013. https://doi.org/10.1016/j.engstruct.2013.10.030 